\title{
DYNAMICS OF A DISORIENTED CHIRAL CONDENSATE
}

\author{
Jean-Paul BLAIZOT ${ }^{a \nsupseteq}$ and André KRZYWICKI ${ }^{b}$ \\ ${ }^{a}$ Service de Physique Théorique, Centre d'Etudes de Saclay, \\ F-91191 Gif-sur-Yvette, France \\ ${ }^{b}$ Laboratoire de Physique Théorique et Hautes Energies, Bât. 211, \\ Université de Paris-Sud, F-91405 Orsay, France
}

\begin{abstract}
We use the linear $\sigma$ model to analyse the dynamics of a disoriented chiral condensate. For idealized boundary conditions appropriate to high energy collisions, the problem can be reduced to a one dimensional one. The evolution of the chiral state is then that of a simple dynamical system and can be studied analytically.

PCAC number(s): 25.75.+r, 13.85.Hd , 03.50.Kk
\end{abstract}

February 1993

LPTHE Orsay 94/18

SPhT T94/019

\footnotetext{
${ }^{1}$ Electronic mail: blaizot@amoco.saclay.cea.fr

${ }^{2}$ Electronic mail: krz@qcd.th.u-psud.fr

${ }^{3}$ Laboratoire asocié au C.N.R.S.
} 


\section{INTRODUCTION}

The aim of this paper is to present a simple, analytical discussion of the time evolution of a disoriented chiral condensate (DCC) which could be produced in a high-energy collision [1]-[3]. We shall extend our previous investigation [3] and make contact with recent works which have been triggered by the idea of a "quench", put forward in ref. [4]. Specifically, we shall study the classical equations of motion of the linear $\sigma$ model, assuming random initial conditions for the fields and their derivatives. This randomness could result from a sudden cooling of a hot quark-gluon plasma, as proposed in [4], but we shall not insist on an interpretation based on initial thermal equilibrium. The evolution of the system will be described by the classical equations of motion of the $\sigma$ model. We neglect possible quantum effects (for a recent discussion of the decoherence of the radiation off colliding heavy ions see [5]). The main motivation for using the linear, instead of the non-linear $\sigma$ model as in [3], is to allow for the possibility to start the evolution from a chirally symmetric random configuration, as in [4]. As we shall see, however, many features of our previous study survive.

As in ref. [3] we adopt the idealization originaly proposed by Heisenberg [6]: we assume that at time $t=0$ the whole energy of the collision is localized within an infinitesimally thin slab with infinite transverse extent. The symmetry of the problem then implies that the fields depend only on the invariant $\tau=\sqrt{t^{2}-x^{2}}$, where $x$ is the longitudinal coordinate. This is of course a dramatic simplification of the actual situation. In particular, our assumptions lead to unrealistic correlations both in the transverse plane and in rapidity. However, with this idealization the problem becomes $1+1$ dimensional, and can be treated analytically. This will allow us to identify clearly various regimes of the dynamics of chiral condensates in the presence of the longitudinal expansion which necessarily occurs in case of high energy collisions. We believe that such analytical studies are complementary to numerical simulations [4, 7, 8] aiming at getting a more realistic picture, and they may prove useful in understanding the results obtained in such simulations.

Although we use Heisenberg's idealization to justify working in $1+1$ dimensions, we restrict our discussion to proper times $\tau>\tau_{0}$. An extension of the model down to $\tau=0$ leads to unphysical results and is completely unjustified. Heisenberg [6] has pointed out that in a wide class of non-linear 
theories with gradient interactions, the fields have to vanish like $\tau$ in the limit $\tau \rightarrow 0$. It seems more likely, however, that the fields $(\vec{\pi}, \sigma)$ do not tend to any well defined limit as $\tau \rightarrow 0$ but behave chaotically (in the loose sense of the word). In any case, as $\tau \rightarrow 0$, the relevant degrees of freedom are presumably no longer hadronic and, besides, the effective theory is certainly more complicated than the linear $\sigma$ model.

Thus, we shall assume that starting from some finite time $\tau_{0}$, we can describe the state of matter produced in a collision by the linear $\sigma$ model. Our results will turn out to be little dependent of the specific value of this time $\tau_{0}$, as long as it is chosen so that at $\tau_{0}$ the system is in the chirally symmetric configuration. The values of the fields at this time are taken as random variables. In fact, as we shall show in section 2 , the equations of motion are, for short times, equivalent to the conservation of the isospin vector and axial vector currents. The bulk of the dynamics will be entirely determined by the values of these currents at $\tau_{0}$. In section 3 , we shall identify several regimes of the evolution of our chiral condensate. We shall see that the problem reduces to that of finding the motion of a fictitious particle in a time dependent potential and subject to a time dependent friction. The paper ends in section 4 by a discussion.

\section{FORMULATION OF THE MODEL}

Our system is described by the Lagrangian of the linear $\sigma$ model, with the standard chiral symmetry breaking term:

$$
L=\frac{1}{2}\left[(\partial \sigma)^{2}+(\partial \vec{\pi})^{2}\right]-\frac{\lambda}{4}\left(\sigma^{2}+\vec{\pi}^{2}-1\right)^{2}+H \sigma .
$$

The fields $\sigma$ and $\vec{\pi}$ are dimensionless and the parameters $\lambda$ and $H$ have the dimension of $[\mathrm{mass}]^{2}$. We have scaled out a dimensionless factor $S f_{\pi}^{2}$, which would normally occur in the expression of the Lagrangian for the 3dimensional model [3]. The parameters of the model may be related to the masses of the $\sigma$ and pion field, respectively: $m_{\sigma}^{2} \approx 2 \lambda$ and $m_{\pi}^{2}=H$. For the sake of the argument we shall proceed as if one had $m_{\sigma} \gg m_{\pi}$. We shall return to this point in the discussion.

The equations of motion read 


$$
\tau^{-1}\left(\tau \sigma^{\prime}\right)^{\prime}=-\lambda\left(\sigma^{2}+\pi^{2}-1\right) \sigma+H
$$

and

$$
\tau^{-1}\left(\tau \vec{\pi}^{\prime}\right)^{\prime}=-\lambda\left(\sigma^{2}+\pi^{2}-1\right) \vec{\pi},
$$

where the prime denotes differentiation with respect to $\tau$. From (3) one easily gets

$$
\vec{\pi} \times \vec{\pi}^{\prime}=\frac{\vec{a}}{\tau}
$$

where $\vec{a}$ is an integration constant. This is a direct consequence of the conservation of the isovector current together with the assumption that the fields depend on $\tau$ only. Hence the component of $\vec{\pi}$ along $\vec{a}$ vanishes:

$$
\pi_{a}=0 .
$$

A further consequence of the equations of motion is the equation

$$
\tau^{-1}\left[\tau\left(\vec{\pi} \sigma^{\prime}-\sigma \vec{\pi}^{\prime}\right)\right]^{\prime}=H \vec{\pi},
$$

which is just the statement of partial conservation of the isoaxial-vector current (PCAC).

For small enough $\tau$, the effect of the symmetry breaking term $H \vec{\pi}$ can be neglected. The isoaxial-vector current is then conserved, and one can write, in analogy to (來)

$$
\vec{\pi} \sigma^{\prime}-\sigma \vec{\pi}^{\prime}=\frac{\vec{b}}{\tau}
$$

where $\vec{b}$ is another integration constant. When eqs. (4) and (17) are satisfied, the component of $\vec{\pi}$ along $\vec{c}=\vec{a} \times \vec{b}$ equals $\sigma$, up to the constant factor $a / b$. The forms of the conservation laws (4) and (7) reveal important features of our model. Because of the symmetry of the fields, and the classical dynamics, in the limit of vanishing pion mass the isospin vector and axial vector currents keep fixed orientations in isospace as the system evolves. Of course, any orientation is equally probable. But once fixed in the initial conditions, it remains constant. 
The equations (4) and (7) are identical to those obtained in the nonlinear $\sigma$ model [3], and they are independent of $\lambda$. The non-linear $\sigma$ model is obtained in the limit $\lambda \rightarrow \infty$. In this limit, the motion takes place on the hypersphere $\sigma^{2}+\vec{\pi}^{2}=1$ and is entirely determined by the conservation laws for the isospin currents. When $\lambda$ is large, but not infinite, one can still separate the general motion into a motion on the sphere, which one may loosely associate with the pion degrees of freedom, and a motion normal to the sphere which involves the $\sigma$ degree of freedom. As long as the parameter $H$ can be neglected, there is no scale controlling the motion on the sphere, while $\lambda^{-1 / 2}$ controls the time dependence of the oscillations normal to the sphere. When $H$ can no longer be ignored, it controls the time behaviour of pionic excitations.

In order to analyse the evolution of the chiral state, we shall use the following parametrisation which takes into account the symmetries of the problem:

$$
\begin{aligned}
\pi_{b} & =r \sin \theta \\
\pi_{c} & =r \cos \theta \sin \omega \\
\sigma & =r \cos \theta \cos \omega .
\end{aligned}
$$

Note that $\sigma^{2}+\pi^{2}=r^{2}$. Our problem has now been reduced to a simple dynamical problem for a point particle with one radial degree of freedom, $r$, correesponding to the $\sigma$ excitations, and two angular degrees of freedom $\theta$ and $\omega$, corresponding to the pionic excitations. As will become clear later, the evolution of the system depends upon the initial conditions mostly via the constants $a$ and $b$ (while the detailed initial values of $r, \theta, \omega$ and their derivatives are not essential). The two constants $a$ and $b$ determine the strength of the isospin vector and axial vector currents (note that due to a different choice of variables, the present $a$ and $b$ are twice as large as those introduced in ref. [3]). They are random variables whose probability distribution will be calculated in the last section.

As a final remark in this section, we note that when eqs. (田) and (7) are satisfied, the angle $\omega$ is constant and given by

$$
\omega=\arctan \frac{a}{b}
$$


Thus the motion is planar in the 3 -space $\left(\sigma, \pi_{b}, \pi_{c}\right)$. This plane becomes almost identical to $\left(\pi_{b}, \sigma\right)$ when $b \gg a$. We shall find convenient, at some point in the forthcoming discussion, to work in this particular limit.

\section{DCC AS A SIMPLE DYNAMICAL SYS- TEM}

The initial time $\tau_{0}$ is supposed to be such that the mass term $\propto H$ can be ignored at the beginning of the evolution. Then the initial motion is governed by the two conservation laws for the isospin currents, i.e. eqs. (4) and (7). These translate into the following equation for the "angular momentum" of the fictitious point particle associated with our system:

$$
r^{2} \theta^{\prime}=\frac{\kappa}{\tau}
$$

The equation for the radial motion is obtained by neglecting $H$ in the equations of motion (2)-(3), multiplying the equation for $\sigma\left(\pi_{b}\right)$ by $\cos \theta(\sin \theta)$, and adding the resulting equations. We get

$$
r^{\prime \prime}+\frac{1}{\tau} r^{\prime}=\frac{\kappa^{2}}{\tau^{2} r^{3}}-\lambda r\left(r^{2}-1\right) .
$$

We have used eq. (10) to eliminate $\theta^{\prime}$, and also the fact that $\omega$ is constant when the isospin currents are strictly conserved. The second term on the left-hand side may be interpreted as a time dependent friction. Thus, the fictitious particle experiences damped oscillations. The amplitude of these oscillations can be initiallly quite important if $r\left(\tau_{0}\right)$ is small. In that case, the repulsive force represented by the first term on the right-hand side of (11) provides to the particle a large velocity. However, the oscillations are rapidly damped, while the repulsive force itself decays fast with time.

Further insight on the radial motion can be gained by considering the time variation of the mechanical energy of the fictitious particle. This is given by

$$
\frac{d}{d \tau}\left[\frac{\left(r^{\prime}\right)^{2}}{2}+\frac{\lambda}{4}\left(r^{2}-1\right)^{2}+\frac{\kappa^{2}}{2 \tau^{2} r^{2}}\right]=-\frac{\kappa^{2}}{r^{2} \tau^{3}}-\frac{\left(r^{\prime}\right)^{2}}{\tau}<0 .
$$


This derivative is particularly large, in absolute value, during the very early stage of the evolution due to the presence of the term $\sim \tau^{-3}$. The whole damping is of kinematical origin; it reflects the decrease of the field energy density as the system expands. One observes that the influence of the angular motion on the radial motion quickly disappears. The energy in the angular motion is controlled by $\kappa$. It becomes of the same order of magnitude as the potential energy $\propto \lambda$ when $\tau \sim \kappa / \sqrt{2 \lambda}$, and is negligible at later times.

For not too small $\tau$ it is certainly a good approximation to linearize the expression appearing on the right-hand side of (11). There results an inhomogeneous Bessel equation for $r-1$. The solution takes a particularly suggestive form for $\sqrt{2 \lambda} \tau \gg \max \left[\kappa^{2}, 1\right]$ :

$$
r \approx 1+\frac{C}{(\sqrt{2 \lambda} \tau)^{\frac{1}{2}}} \cos (\sqrt{2 \lambda} \tau+\eta),
$$

where $C$ and $\eta$ are integration constants fixed by the initial conditions. Thus, because of the damping caused by the expansion, the system is driven towards the orbit $r=1$ [strictly speaking, for $H \neq 0$, the orbit would rather be at $r=1+O(H / \lambda)]$.

When $r \approx 1$, the integration of eq. (10) yields $\theta \approx \kappa \ln \left(\tau / \tau_{0}\right)$ and one recovers the solution of the non-linear $\sigma$ model presented in ref. [3]. In fact the approach to the orbit is fairly slow, and a change of regime will in most cases take place before the orbit is actually reached. The oscillations in the radial motion have a large frequency, $\propto \sqrt{2 \lambda}$. Since the angular motion, controlled by $\kappa$, is generically slower than the radial one, one can, with a good accuracy, replace $r^{2}$ in eq. (10) by its expectation value over a few periods of the radial motion. This expectation value reaches a value $\approx 1$ in a short time. Therefore, the result $\theta \approx \kappa \ln \left(\tau / \tau_{0}\right)$ can be taken as a good approximation from nearly the beginning of the evolution.

A change of regime is expected to occur when the mass term $\propto H$ can no longer be ignored. Let us, therefore, discuss in more detail the quality of the approximation $H=0$. Integrating eq. (6) for $\pi_{b}$ one has

$$
\tau\left(\pi_{b} \sigma^{\prime}-\sigma \pi_{b}^{\prime}\right)=b+H \int^{\tau} \tau r \sin \theta d \tau .
$$

In estimating the integral above it is important to notice that the integrand is oscillating, and it does so quite rapidly when $\kappa$ is large. In order to find a rough estimate, we replace again $r$ by 1 , its average value, so that 
$\theta=\kappa \ln \left(\tau / \tau_{0}\right)$ (we assume $\theta\left(\tau_{0}\right)=0$ to avoid the proliferation of inessential integration constants). The integral in (14) is then easily evaluated. For large $\kappa$, it equals $\left(\tau^{2} / \kappa\right) \cos \left(\kappa \ln \left(\tau / \tau_{0}\right)\right.$. Therefore, the neglect of $H$ is justified as long as

$$
\tau^{2} \ll \frac{b \kappa}{H} .
$$

It remains to determine the conditions ensuring that $\omega=$ const., because the planarity of the motion in the 3 -space $\left(\sigma, \pi_{b}, \pi_{c}\right)$ is another characteristic feature of the early, chirally symmetric regime. Using the polar parametrisation (8) and the equations (6) and (4) one finds after some algebra

$$
\cos \theta \sin \left(\omega-\omega_{0}\right)=\frac{H}{\kappa}\left[\cos \theta \sin \omega \int^{\tau} \tau r \sin \theta d \tau-\sin \theta \int^{\tau} \tau r \cos \theta \sin \omega d \tau\right]
$$

where $\omega_{0}=\arctan (a / b)$, the value $\omega$ takes when $H=0$. On the right-hand side set $\omega=\omega_{0}+\delta \omega$ and expand neglecting terms of $\mathrm{O}\left[(\delta \omega)^{2}\right]$. Furthermore, set again $r=1$ and $\theta=\kappa \ln \left(\tau / \tau_{0}\right)$ to get

$$
\cos \theta \delta \omega \approx-\frac{H \tau^{2}}{\kappa^{3}}\left[a-b \cos ^{2} \theta\right]-\frac{b H}{\kappa^{2}} \sin \theta \int^{\tau} \tau \cos \theta \delta \omega d \tau
$$

One can see that, as long as $\cos \theta$ is not small, $|\delta \omega|$ is of order $H \tau^{2} / \kappa^{2}$. However, when $\cos \theta=0$, the left-hand side of (17) vanishes identically, and a perturbative (in $H$ ) calculation of $\delta \omega$ is impossible. However, one easily sees that the right-hand side of (17) vanishes when the time average $\langle\delta \omega\rangle \approx-a / b$. We conclude that the angle $\omega$ oscillates with an amplitude of order $a / b$, i.e. independent of $H$. We shall assume herefrom that

$$
b \gg a
$$

The constraint (18) makes the problem technically simpler, since $\omega$ remains small and the motion on the orbit is quasi-planar at any time. We do not think that limiting the discussion to this case is a severe restriction. The general case is just more cumbersome, without being really more instructive. With (18) we gain considerably in clarity. Furthermore, only for large enough $b$ the problem is really interesting, a point that will become more and more clear as the discussion will develop. Finally, as will be shown in the last 
section, it is improbable that both $b$ and $a$ are large. When (18) holds, eq. (15) can be rewritten as follows

$$
\tau \ll \frac{b}{\sqrt{H}} .
$$

The presence of $H$ in the equations of motion is an inessential complication as long as (19) is true. We shall see that a dramatic change of regime occurs when $\tau \approx b / \sqrt{H}$.

Consider first what happens at large times. To this end we use eqs. (4) and (6). Working in the polar parametrization (8), with $r=1$, one easily convinces oneself that $\theta, \omega \rightarrow 0$ like some inverse power of $\tau$ when $\tau \rightarrow \infty$, modulo possible oscillations. For small enough $\theta, \omega$ eqs. (6) read

$$
\begin{aligned}
\tau^{-1}\left(\tau \theta^{\prime}\right)^{\prime} & =-H \theta \\
\tau^{-1}\left(\tau \omega^{\prime}\right)^{\prime} & =-H \omega,
\end{aligned}
$$

while eq. (4) becomes a constraint on the Wronskian $W(\omega, \theta)$ :

$$
W(\omega, \theta)=\frac{a}{\tau}
$$

Recall that the operator appearing on the left-hand side of eqs. (20) is the form taken by $\partial^{2}$ acting on a field depending on $\tau$ alone. Furthermore, in the regime that we are now considering, $\theta \approx \pi_{b}$ and $\omega \approx \pi_{c}$. Hence, eqs. (20) describe the free propagation of massive pions, the mass being $H^{\frac{1}{2}}$, as expected. Eqs. (20) become Bessel equations when the variable $\sqrt{H} \tau$ is used. One has

$$
\begin{aligned}
\theta & \approx \frac{D}{(\sqrt{H} \tau)^{\frac{1}{2}}} \cos (\sqrt{H} \tau+\zeta) \\
\omega & \approx \frac{a}{D(\sqrt{H} \tau)^{\frac{1}{2}}} \sin (\sqrt{H} \tau+\zeta),
\end{aligned}
$$

for $\sqrt{H} \tau \gg 1$, where $D$ and $\zeta$ are integration constants. Notice, that the amplitudes and phases of $\theta$ and $\omega$ are related by the constraint (21). Eqs. (22) hold asymptotically, independently of the values taken by the constants $a$ and $b$. 
In order to determine the constant $D$ one needs to match the two extreme regimes discussed so far, which is not an easy task if attempted in full generality but which simplifies considerably when $a \ll b$.

By using the approximation already discussed in which one replaces $r$ by its expectation value, and $r r^{\prime}$ by zero, and assuming that $\omega$ remains small enough, we can transform eq. (6) for $\pi_{b}$ into

$$
\theta^{\prime \prime}+\frac{1}{\tau} \theta^{\prime}+H \sin \theta=0
$$

This is the equation of a pendulum subject to a time dependent friction. The asymptotic solution, for $\tau \rightarrow \infty$, has already been written in eq. (22) and describes an oscillatory motion. The initial value of $\theta$ is not very important. However, for large $b$, the derivative $\theta^{\prime}=b / \tau$ remains large, generically, at the time when the expectation value of $r$ becomes close to unity. As long as the angular velocity is large enough, the motion is circular. Our purpose, now, is to discuss the transition from the circular to oscillatory motion of this simple dynamical system. In particular, we shall determine the time when the transition takes place and the amplitude of final oscillations (the parameter $D$ in eqs. (22)).

Eq. (23) implies that

$$
\frac{d}{d \tau} E(\tau) \equiv \frac{d}{d \tau}\left[\frac{1}{2}\left(\theta^{\prime}\right)^{2}+H(1-\cos \theta)\right]=-\frac{1}{\tau}\left(\theta^{\prime}\right)^{2}<0 .
$$

Thus the "energy" $E(\tau)$ of the pendulum is a strictly decreasing function of time. As long as the total energy is much larger than the potential energy $H(1-\cos \theta)$, the motion is circular. The transition occurs, when $E(\tau)$ "hits" the potential. Let us solve eq. (23), treating this potential as a perturbation. One finds

$$
\theta \approx b \ln \left(\tau / \tau_{0}\right)
$$

and

$$
\theta^{\prime} \approx \frac{b}{\tau}+H \frac{\tau}{b} \cos \left[b \ln \left(\tau / \tau_{0}\right)\right]
$$

Since the slope of $E(\tau)$ decreases rapidly with $\tau$, for large $b$ the potential is "hit" near its top at time $\tau$ satisfying $\cos \left[b \ln \left(\tau / \tau_{0}\right)\right] \approx-1$. The transition time is estimated by setting $\theta^{\prime}=0$ and is found to be 


$$
\tau_{\text {trans }} \approx \frac{b}{\sqrt{H}}, \quad b \gg 1 .
$$

As one might have expected, the transition occurs when the naively estimated kinetic energy becomes comparable to the potential energy. The amplitude of oscillation that results is, of course bounded by $\pi$. Actually, it is close to $\pi$ just after the transition, precisely because the potential is "hit" near its top. Linearizing the potential one gets the solution (22). Keeping in mind the remark just made about the amplitude, and using (27) one finds

$$
D \approx \operatorname{const} . \sqrt{b}, \quad b \gg 1,
$$

where the constant is of order unity. The amplitude of the pion field is $\propto \sqrt{b}$ and, consequently, the height of the rapidity plateau is $\propto b$. This is similar to the result found in [3] in the framework of the non-linear $\sigma$ model. As was the case there, the parameter $b(\approx \kappa$ when $a \ll b)$ is a measure of the energy released in the decay of the condensate.

\section{DISCUSSION}

We have identified several stages in the evolution of the chiral condensate. First there is a short phase in which angular motion and radial motion are strongly coupled. This phase lasts for a time $\sim b / \sqrt{2 \lambda}$. Next, the fictitious particle rotates slowly, while oscillating rapidly about the equilibrium radial position $r \approx 1$. Because of the expansion of the physical system, the energy of the fictitious particle is damped. At some point there is a transition from circular to oscillatory motion, which actually corresponds to free propagation of final state pions. The transition takes place when the mass of pion excitation can no longer be neglected, and it occurs typically when $\tau \sim b / \sqrt{H}$.

The pion field has a random orientation in isospace and therefore the fraction $f$ of neutral pions is distributed according to the law

$$
d P(f)=\frac{1}{2 \sqrt{f}} d f
$$

written first explicitly in ref. [3] (but obtained earlier, and independently, by Bjorken [9]). At all times the system is subject to "friction". The latter is of 
purely kinematical origin and reflects the boost invariance imposed on our solution. There is no true energy dissipation. Simply, the expansion causes the field energy in a covolume to decrease.

The two time scales $\tau_{1}=(2 \lambda)^{-\frac{1}{2}}$ and $\tau_{2}=(H)^{-\frac{1}{2}}$, if estimated using the phenomenological values of the pion and $\sigma$ masses, differ only by a factor of 4. This is presumably not sufficient to guarantee a clean separation of regimes, especially when $b$ is not large enough (and may be related to difficulties in producing large domains of misaligned vacuum reported in ref. [0]). Although it seems that the picture becomes sharper when the condensate carries more energy, there is a price to be paid for that: as will be shown in a moment, the probability that $b$ is large falls exponentially.

Let the fields $(\vec{\pi}, \sigma)$ and their (proper) time derivatives be Gaussian random variables with variances $\sigma_{1}$ and $\sigma_{2}$, respectively, at some initial time $\tau_{0}$ belonging to the chiral symmetric regime (cf. ref. [1] ). With this assumption let us calculate the probability $P(\kappa, \omega)$ to find a given value of $\kappa=\sqrt{a^{2}+b^{2}}$ and $\omega=\arctan (a / b)$. Using the polar parametrization of the fields in the randomly oriented hyperplane $\perp \vec{a}$, together with the constraint $\kappa=\tau_{0} r^{2} \theta^{\prime}$ (cf. (10)), one finds, after a straightforward calculation:

$$
\frac{d^{2}}{d \kappa d \omega} P(\kappa, \omega)=\frac{\kappa}{2\left(\pi \sigma_{1} \sigma_{2} \tau_{0}\right)^{2}} K_{1}\left(\frac{\kappa}{\sigma_{1} \sigma_{2} \tau_{0}}\right),
$$

where $K_{1}(z)$ denotes the second modified Bessel function, $K_{1}(z) \sim \sqrt{\pi / 2 z} e^{-z}$ for $z \rightarrow \infty$. The probability distribution (30) falls exponentially at large $\kappa$. This result is unlikely to be of much phenomenological relevance, since it is obtained in a $1+1$ dimensional model. The reduction to $1+1$ dimensions means full translational symmetry in transverse coordinates and, in particular, coherence over large transverse distance. The probability that such coherence does occur cannot be estimated within the framework of the model. Eq. (30) represents nevertheless a significant conceptual progress with respect to the discussion of refs. [1] - [3]: the present approach, which has been partly inspired by ref. [四], enables one to determine the absolute probability of observing a disoriented chiral condensate with given global characteristics.

The initial randomness of the fields $(\vec{\pi}, \sigma)$, and their derivatives, does not necessarily reflect thermalization occuring at the early stage of the collision process. Actually, the most interesting aspect of the quench model put for- 
ward, in this context, by Rajagopal and Wilczek is the out of equilibrium evolution of the system. In discussing this evolution one does not need to talk about temperature at all. Since the seminal work of Landau [10], the study of complicated nuclear collisions uses the concept of (local) thermal equilibrium as the central piece of the paradigm. If we were to assume that local thermal equilibrium is maintained throughout the time development, we would be led to use hydrodynamical evolution equations. The resulting evolution would be very different from that obtained here. It is unclear, at this moment, whether the disoriented chiral condensate can be really produced in high energy collisions. However, the theoretical investigation of this as yet undiscovered phenomenon has already turned out to be useful, since

it has been a good pretext for trying to figure out non-equilibrium aspects of high-energy nuclear collisions.

\section{ACKNOWLEDGEMENTS}

An interesting conversation with A. Białas and a helpful suggestion from J. Ginibre are acknowledged. 


\section{References}

[1] A.A. Anselm, Phys. Lett. B 217 ,169 (1989); A.A. Anselm and M. Ryskin, Phys. Lett. B 266, 482 (1991).

[2] J.D. Bjorken, Int. J. Mod. Phys. A 7, 4819 (1992) Acta Phys. Polon. B 23, 561 (1992).

[3] J.P. Blaizot and A. Krzywicki, Phys. Rev. D 46, 246 (1992).

[4] K. Rajagopal and F. Wilczek, Nucl. Phys. B 399, 395 (1993); Nucl. Phys. B 404, 577 (1993).

[5] A. Krzywicki, Phys. Rev. D 48, 5190 (1993).

[6] W. Heisenberg, Z. Phys. 133, 65 (1952).

[7] S. Gavin, A. Gocksch and R.D. Pisarski, Brookhaven Report No. BNLGGP-1 (October 1993).

[8] Z. Huang and X.N. Wang, Berkeley Report No. LBL-34931 (December 1993).

[9] J.D. Bjorken, Inv. talk at Symposium on the SSC Laboratory, Report No. SLAC-PUB-5673 (October 1991).

[10] L.D. Landau, Izv. Akad. Nauk SSSR, Ser. Fiz. 17, 51 (1953). 